Enfermagem Brasil 2018;17(3);227-35

\title{
ARTIGO ORIGINAL \\ Características sociodemográficas e clínicas da hanseníase: um estudo populacional
}

Emanuelle Malzac Freire de Santana*, Karen Krystine Gonçalves de Brito*, Ester Missias Villaverde Antas**, Smalyanna Sgren da Costa Andrade*, Irkatania Vitorino Diniz*, Siméia de Macêdo Lima***, Mirian Alves da Silva, D.Sc. ${ }^{* \star *}$

*Doutoranda, Programa de Pós-Graduação em Enfermagem, Universidade Federal da Paraíba, João Pessoa/PB, **Mestranda, Programa de Pós-Graduação em Enfermagem, Universidade Federal da Paraíba, João Pessoa/PB, ${ }^{* * *}$ Graduanda em Enfermagem, Universidade Federal da Paraíba, João Pessoa/PB, ${ }^{* * \star * P r o f e s s o r a ~ D e p a r t a m e n t o ~ d e ~ E n f e r m a g e m ~ C l i ́ n i c a, ~ U n i v e r s i d a d e ~}$ Federal da Paraíba, João Pessoa/PB

Recebido em 11 de julho de 2017; aceito em 26 de dezembro de 2017.

Endereço para correspondência: Emanuelle Malzac Freire de Santana, Rua Julieta Marinho Marsicano, 109/204, 58035-310 João Pessoa PB, E-mail: manumalzac@gmail.com; Karen Krystine Gonçalves de Brito: karen_enf@yahoo.com.br; Ester Missias Villaverde Antas: ester_villaverde@yahoo.com.br; Smalyanna Sgren da Costa Andrade: nana_sgren@hotmail.com; Irkatania Vitorino Diniz: iraktania@hotmail.com; Siméia de Macêdo Lima: simeiamacedo@windowslive.com; Mirian Alves da Silva: miads.enf@gmail.com

\section{Resumo}

Introdução: Apesar da redução nos coeficientes de prevalência e de detecção de casos novos, a hanseníase ainda se configura como problema de saúde pública no Brasil. Objetivo: Descrever o perfil sociodemográfico e clínico de pacientes com hanseníase; e verificar a associação entre suas características clínicas segundo os anos de 2009 a 2014 . Material e métodos: Estudo descritivo, retrospectivo, populacional e documental, com delineamento quantitativo desenvolvido de 2009 a 2014 em centro de referência em João Pessoa/PB. Envolveu 414 prontuários, utilizando-se formulário estruturado para coleta de dados contemplando variáveis sociodemográficas, clínicas e da avaliação neurológica simplificada no diagnóstico e na alta. Resultados: Observou-se predominância de homens $(58,7 \%)$, faixa etária de 31 a 45 anos $(27,8 \%)$, baixa escolaridade $(65,9 \%)$, tipo multibacilar $(60,6 \%)$, forma dimorfa $(35 \%)$, grau 0 de incapacidade $(59,4 \%)$, pés comprometidos $(37,2 \%)$ e sem nervos afetados $(52,4 \%)$. Entre $2009-2014$ constatou-se diferença significativa para o grau de incapacidade $(\mathrm{p}=0,038)$, com aumento do número de casos diagnosticados com grau 0 ; para o sítio corporal olho $(p=0,004)$ e mão $(p=0,003)$ com diminuição de seu acometimento; e para os nervos $(p=0,020)$ com aumento de indivíduos sem nervos afetados. Conclusão: Os resultados indicam que apesar da hanseníase permanecer ativa na região estudada, a assistência tem se mostrado satisfatória.

Palavras-chave: hanseníase, epidemiologia, prevenção e controle, atenção secundária à saúde.

\section{Abstract \\ Sociodemographic and clinical characteristics of leprosy: a population study}

Introduction: Despite the reduction in the prevalence and detection coefficients of new cases, leprosy is still public health problem in Brazil. Aim: To describe the sociodemographic and clinical profile of patients with leprosy; and to verify the association between its clinical characteristics according to the years 2009 to 2014. Methods: Descriptive, retrospective, population and documentary study, with a quantitative delineation developed from 2009 to 2014 in reference center in João Pessoa/PB. This study involved 414 medical records, using structured form for data collection contemplating sociodemographic and clinical variables and simplified neurological evaluation in diagnosis and discharge. Results: We observed a predominance of men (58.7\%), age group $31-45$ years $(27.8 \%)$, low schooling $(65.9 \%)$, multibacillary type $(60.6 \%)$, dimorphous $35 \%)$, degree of disability $(59.4 \%)$, impaired feet (37.2\%) and no affected nerves (52.4\%). From 2009 to 2014, there was significant difference for the degree of disability $(p=0.038)$, with an increase of cases diagnosed with grade 0 ; to the body eye site $(p=0.004)$ and hand $(p=0.003)$ with decrease of its involvement; and for the 
nerves $(p=0.020)$ with increase of individuals without affected nerves. Conclusion: The results indicate that although leprosy remains active in the studied region, care strategy has been satisfactory.

Key-words: leprosy, epidemiology, prevention and control, secondary care.

\section{Resumen \\ Características sociodemográficas y clínicas de la lepra: un estudio poblacional}

Introducción: A pesar de la reducción en los coeficientes de prevalencia y de detección de casos nuevos, la lepra aún se configura como problema de salud pública en Brasil. Objetivo: Describir el perfil sociodemográfico y clínico de pacientes con lepra; y verificar la asociación entre sus características clínicas según los años 2009 al 2014. Material y métodos: Estudio descriptivo, retrospectivo, poblacional y documental, con delineamiento cuantitativo desarrollado entre 2009 y 2014 en centro de referencia en João Pessoa/PB. Se evaluaron 414 historias clínicas, utilizando formulario estructurado para colección de datos contemplando variables sociodemográficas, clínicas y de evaluación neurológica simplificada en el diagnóstico y en el alta hospitalaria. Resultados: Se observó predominancia de hombres $(58,7 \%)$, grupo de edad $31-45$ años $(27,8 \%)$, baja escolaridad $(65,9 \%)$, tipo multibacilar $(60,6 \%)$, forma dimorfa (35\%), grado 0 de incapacidad $(59,4 \%)$, pies comprometidos $(37,2 \%)$ y sin nervios afectados $(52,4 \%)$. Entre $2009-2014$ se constató una diferencia significativa para el grado de incapacidad $(p=0,038)$, con aumento del número de casos diagnosticados con grado 0 ; para el sitio corporal ojo $(p=0,004)$ y mano $(p=0,003)$ con disminución de su afectación; y para los nervios $(p=0,020)$ con aumento de individuos sin nervios afectados. Conclusión: Los resultados indican que a pesar de la lepra permanecer activa en la región estudiada, la asistencia se ha mostrado satisfactoria.

Palabras-clave: lepra, epidemiología, prevención y control, atención secundaria de salud.

Introdução

Nas últimas décadas, ocorreu redução global da prevalência da hanseníase de mais de 5 milhões de casos em 1980 para menos de 200 mil em 2015. Todavia, apesar dos avanços para o controle da endemia, a hanseníase ainda se mantém como relevante problema de saúde pública no Brasil [1], ocupando a segunda colocação em número de notificações no ranking mundial, atrás apenas da Índia. Em 2015, foram notificados 26.395 novos casos representando $13 \%$ de todas as notificações da doença no mundo [2].

Apesar da redução contínua nos coeficientes de prevalência e de detecção de casos novos ao longo dos anos, o país ainda não atingiu a meta de eliminação proposta pela Organização Mundial de Saúde (OMS), representada pela redução do coeficiente de prevalência a menos de um caso em cada dez mil habitantes. Em 2012, a prevalência no país foi de 1,51/10.000 habitantes [3].

No Brasil, a distribuição dos casos de hanseníase não é homogênea [4] e existem regiões que ainda possuem alto padrão de endemicidade, sendo consideradas áreas importantes na manutenção da sua transmissão.

Dentre os Estados brasileiros, a região Nordeste aglomera uma das maiores endemias da doença, sendo responsável por coeficiente de detecção de casos de 22,56/100.000 habitantes em 2015 [5]. Integrante deste cluster, a Paraíba apresenta tendência de queda para o coeficiente desde 2006, com projeções de 18,8/100.000 habitantes para os anos de 20122014 [6]. Em 2015, o coeficiente de detecção de casos foi de 13,17/100.000 habitantes, o que representa estabilização e melhora deste indicador epidemiológico [7].

O município de João Pessoa, capital do Estado da Paraíba, é considerado prioritário e estratégico para eliminação da hanseníase de acordo com o "Plano integrado de ações estratégicas de eliminação da hanseníase, filariose, esquistossomose e oncocercose como problema de saúde pública, tracoma como causa de cegueira e controle das geohelmintíases: plano de ação 2011-2015", lançado com o propósito de erradicar ou reduzir de forma drástica a carga desses agravos e, se tratando da hanseníase, enfatiza o aumento da detecção precoce e da cura dos casos diagnosticados [8].

Sabe-se que os serviços de saúde desempenham papel fundamental no diagnóstico da patologia em sua fase inicial e no tratamento oportuno, responsáveis por contribuir para redução da sua prevalência [9]. Desta forma, estudos de descrição epidemiológica da hanseníase em seus diferentes aspectos tornam-se relevantes para subsidiar os gestores e profissionais de saúde no planejamento e desenvolvimento de ações voltadas para o controle 
da doença, além de contribuir para seu monitoramento. O conhecimento do comportamento epidemiológico também permite identificar as áreas de maior gravidade da doença facilitando o planejamento das intervenções.

É papel da Enfermagem incentivar os indivíduos acometidos pela hanseníase sobre a importância da adesão ao tratamento, além de orientá-los sobre os cuidados a serem realizados para evitar possíveis complicações devido ao acometimento nervoso resultante da evolução da doença.

Diante do exposto, o presente estudo tem como questões norteadoras: Qual o perfil sociodemográfico e clínico apresentado pelos pacientes com hanseníase entre os anos de 2009 a 2014? Quais associações existem entre suas características clínicas segundo os anos de 2009 a 2014 ?

Portanto, o objetivo deste estudo foi descrever o perfil sociodemográfico e clínico de pacientes com hanseníase; e verificar a associação entre suas características clínicas segundo os anos de 2009 a 2014.

\section{Material e métodos}

Trata-se de um estudo de caráter descritivo, retrospectivo, base populacional e fonte documental com delineamento quantitativo realizado entre 2009 e 2014 com prontuários de pacientes com diagnóstico de hanseníase que foram atendidos em um centro de referência especializado para o tratamento da doença no Estado da Paraíba, localizado na cidade de João Pessoa.

A população do estudo foi composta por 485 pacientes da região metropolitana de João Pessoa da qual fazem parte 12 municípios, a saber: Alhandra, Bayeux, Caaporã, Cabedelo, Conde, Cruz do Espírito Santo, João Pessoa, Lucena, Pedras de Fogo, Pitimbu, Rio Tinto e Santa Rita.

Como critérios de inclusão, foram instituídos: prontuários de pacientes da região metropolitana de João Pessoa que iniciaram e concluíram o tratamento para hanseníase no centro de referência e que possuíam preenchida a ficha de avaliação simplificada das funções neurais e complicações tanto no diagnóstico quanto na alta por cura. Estabeleceu-se como critérios de exclusão: prontuários de pacientes com comprometimento cognitivo $(n=4)$; prontuários com informações incompletas $(n=61)$; e prontuários de pacientes que não realizaram o tratamento no tempo preconizado pelo MS $(n=6)$. Desta forma, a população do estudo foi composta por 414 prontuários.

Os dados foram coletados de janeiro a abril de 2016 por meio de um instrumento estruturado a partir dos prontuários dos pacientes, dos quais foram extraídas variáveis sociodemográficas (sexo, idade, grau de escolaridade e ocupação) e clínico epidemiológicas (classificação operacional e forma clínica).

O instrumento também foi composto por informações referentes ao momento do diagnóstico e da alta por cura provenientes da ficha de avaliação simplificada das funções neurais e complicações. A utilização desta ficha é preconizada pelo MS e baseia-se em uma avaliação neurológica simplificada para identificar a gravidade das lesões presentes nos olhos, nariz, mãos e pés [10], sendo parte integrante da avaliação realizada no serviço.

Em cada ficha, foi considerado o grau máximo de incapacidades físicas variando de 0 até 2. A graduação 0 é utilizada quando não há nenhum dano presente nos olhos, mãos e pés, o grau 1 corresponde a diminuição ou perda da sensibilidade e o grau 2 quando há presença de deformidades visíveis devido a hanseníase [11].

Os procedimentos para a coleta de dados incluíram a requisição da lista de prontuários de clientes atendidos no período do estudo ao setor de Vigilância Epidemiológica do Centro de Referência para posterior solicitação destes no Serviço de Arquivo Médico e Estatística, setor responsável por organizar e fornecer os prontuários. Em seguida, foi realizado o preenchimento das informações no instrumento.

Os dados obtidos foram codificados para tabulação no aplicativo Microsoft Excel e em seguida exportados para o software Statistical Package for the Social Science (SPSS) versão 20.0, onde foram analisados através de estatística descritiva (frequência absoluta e percentagem) e inferencial (teste de associação de Qui-Quadrado), utilizando-se nível de significância de $5 \%(p<0,05)$.

No processo de investigação foram adotadas as observâncias éticas contempladas pela Resolução 466/12 do Conselho Nacional de Saúde. O projeto foi aprovado pelo Comitê de 
Ética em Pesquisa da Universidade Federal da Paraíba sob protocolo 443/14, CAAE 34284414.3.0000.5188.

\section{Resultados}

A população do estudo foi composta por 414 prontuários de pacientes com hanseníase distribuídos entre ambos os sexos e média de idade de 42,5 anos. A Tabela I demonstra 0 perfil sociodemográfico desta população, com predominância do sexo masculino $(58,7 \%)$, faixa etária de 31 a 45 anos $(27,8 \%)$, baixa escolaridade $(65,9 \%)$ e procedentes do município de João Pessoa (55,3\%).

Tabela I - Perfil sociodemográfico de pacientes com hanseníase entre os anos de 2009 a 2014 na região metropolitana de João Pessoa $(n=414)$. João Pessoa, 2016.

\begin{tabular}{lll}
\hline Variáveis & N & $\%$ \\
\hline Sexo & 243 & \\
Masculino & 171 & 58,7 \\
Feminino & & 41,3 \\
Faixa etária & 30 & 7,2 \\
<15 anos & 83 & 20,0 \\
16 a 30 anos & 115 & 27,8 \\
31 a 45 anos & 112 & 27,1 \\
46 a 60 anos & 74 & 17,9 \\
>60 anos & & \\
Escolaridade & 51 & 12,3 \\
Sem escolaridade & 222 & 53,6 \\
Ensino fundamental & 112 & 27,1 \\
Ensino médio & 29 & 7,0 \\
Ensino superior & & \\
Procedência & 229 & 55,3 \\
João Pessoa & 72 & 17,4 \\
Santa Rita & 66 & 15,9 \\
Bayeux & 12 & 2,9 \\
Lucena & 8 & 1,9 \\
Conde & 6 & 1,4 \\
Cabedelo & 5 & 1,2 \\
Pitimbú & 16 & 4,0 \\
Outros* & & 5
\end{tabular}

Fonte: Dados da pesquisa, 2016; *Refere-se as cidades de Cruz do Espírito Santo, Alhandra, Rio Tinto, Caaporã e Pedras de Fogo.

Na tabela II está descrito o perfil clínico dos participantes, com destaque para a maior concentração da classificação operacional multibacilar (60,6\%), forma clínica dimorfa (35\%), GIF $0(59,4 \%)$, pé como o sítio corporal mais comprometido $(37,2 \%)$ e sem nervos afetados $(52,4 \%)$.

Ao verificar a associação entre o perfil clínico segundo os anos de 2009 a 2014 (Tabela III), constata-se diferença estatisticamente significativa para o GIF $(p=0,038)$ com aumento do número de casos diagnosticados com GIF 0 e redução expressiva do GIF 2 ao longo dos anos. Para os sítios corporais, foi encontrada diferença estatística significativa para os olhos $(p=$ $0,004)$, com aumento do número de indivíduos sem nenhum comprometimento e para as mãos $(p=0,003)$, com diminuição considerável de acometimento, embora no período estudado tenham ocorrido oscilações.

Também se observa diferença estatisticamente significativa $(p=0,020)$ para 0 acometimento dos troncos nervosos com aumento gradativo de indivíduos sem nenhum nervo afetado no período de tempo estudado e redução daqueles com 2 ou mais nervos afetados (Tabela III). 
Tabela II - Perfil clínico de pacientes com hanseníase entre os anos de 2009 a 2014 na região metropolitana de João Pessoa $(n=414)$. João Pessoa, 2016.

\begin{tabular}{lll}
\hline Variáveis & $\mathbf{N}$ & $\%$ \\
\hline Classificação operacional & 163 & 39,4 \\
Paucibacilar & 251 & 60,6 \\
Multibacilar & & \\
Forma clínica & 32 & 7,7 \\
Indeterminada & 120 & 29,0 \\
Tuberculóide & 145 & 35,0 \\
Dimorfa & 92 & 22,2 \\
Virchoviana & 10 & 2,4 \\
Neural pura & 15 & 3,6 \\
Não classificada & & \\
GIF & 246 & 59,4 \\
0 & 122 & 29,5 \\
1 & 46 & 11,1 \\
2 & & \\
Sítio corporal afetado & 28 & 6,8 \\
Olho & 47 & 11,4 \\
Nariz & 58 & 14,0 \\
Mão & 154 & 37,2 \\
Pé & & \\
No de nervos afetados & 217 & 52,4 \\
Nenhum & 68 & 16,4 \\
Um & 129 & 31,2 \\
Dois ou + & &
\end{tabular}

Tabela III - Associação das características clínicas de pacientes com hanseníase segundo os anos de 2009 a 2014 na região metropolitana de João Pessoa $(n=414)$. João Pessoa, 2016.

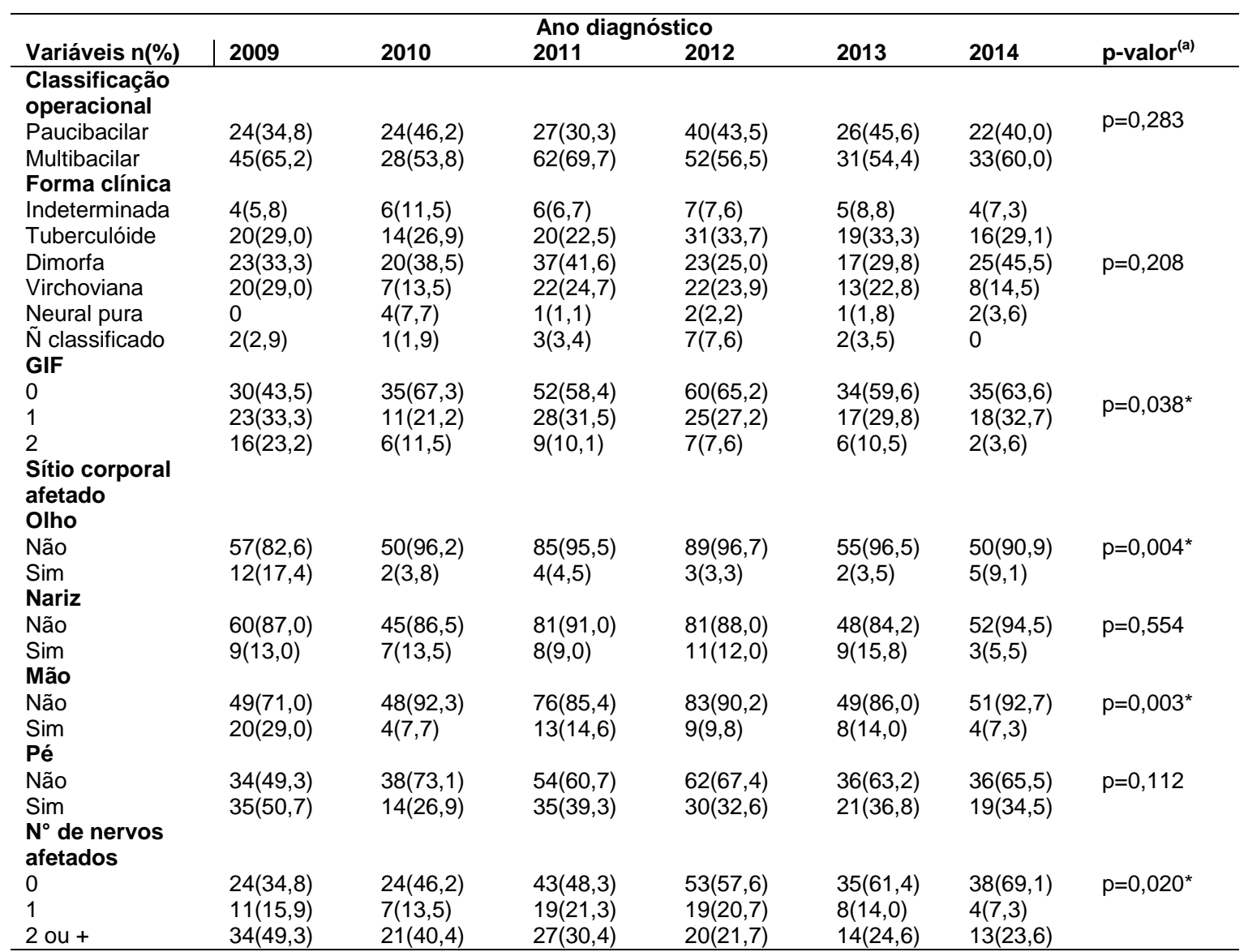

(a)Teste de associação de Qui-Quadrado; Resultado significativo: ( $\left.{ }^{*}\right)$ p-valor < 0.05; Fonte: Dados da pesquisa, 2016. 
O presente estudo permitiu identificar o comportamento sociodemográfico e clínico de pacientes acometidos pela hanseníase na região metropolitana de João Pessoa/PB, sendo este agravo predominante em homens [12-13], faixa etária economicamente ativa [13], baixa escolaridade [13], classificação operacional multibacilar [15], forma clínica dimorfa [15], GIF 0 [16], os pés como o sítio corporal mais comprometido [17] e sem nervos afetados [18]. Estes achados também foram relatados em outros estudos.

O maior percentual de acometimento da doença encontrado no sexo masculino reflete o estilo de vida adotado por essa população que possui frequente exposição a ambientes de risco, o que pode contribuir para elevar o número de casos [19]. Dados da Organização Mundial de Saúde mostram que na maior parte do mundo os homens são afetados mais frequentemente que as mulheres, muitas vezes na proporção de 2:12 [20].

O comprometimento de indivíduos na faixa etária economicamente ativa pode interferir na realização das atividades diárias e ocasionar perdas financeiras e sociais, com impacto sobre a dinâmica econômica familiar devido ao alto poder incapacitante da doença [19,21]. Esses dados são indicadores preocupantes não apenas concernente à saúde pública brasileira, mas também as questões políticas e previdenciárias.

No que diz respeito a escolaridade, o baixo nível de instrução dos pacientes pode contribuir para a difusão da doença devido a possibilidade de interferência na compreensão das informações veiculadas sobre a mesma [21], além de retardar a busca pelos serviços de saúde [22], tornando esta população mais vulnerável ao desenvolvimento de complicações.

Nesta perspectiva, ratifica-se a importância da educação em saúde para sensibilizar as pessoas sobre os primeiros sinais da doença, o diagnóstico precoce e a prevenção de incapacidades [23], posto que indivíduos informados estarão mais propensos a buscarem os serviços de saúde (detecção passiva de casos), aumentando a probabilidade de diagnóstico precoce.

No que tange as características clínicas, estudo realizado em cidade endêmica do norte de Minas Gerais aponta que o indivíduo multibacilar possui chance nove vezes maior em desenvolver algum GIF quando comparado ao paucibacilar [24], o que pode ser justificado pela maior carga bacilar e dano neural presente nesses pacientes [25], influenciando na evolução mais rápida da doença [26].

Considerando que o aparecimento de incapacidades está relacionado ao tempo de evolução da doença [27], os elevados percentuais de casos apresentando as formas clínicas dimorfa e virchowiana, sugerem que o diagnóstico da hanseníase está ocorrendo de forma tardia, o que pode predispor a maior transmissibilidade e consequente instalação de sequelas físicas.

Entretanto, apesar da população ter sido predominantemente multibacilar e, portanto, apresentar maior probabilidade para o desenvolvimento de incapacidades, os achados não foram proporcionais. De acordo com os dados, $60,6 \%$ dos indivíduos foram classificados como multibacilares, todavia $47,6 \%$ apresentaram um ou mais nervos afetados.

Este achado aponta a possibilidade da ocorrência de neurite silenciosa, posto que o comprometimento nervoso pode se desencadear sem dor ou hipersensibilidade a palpação, mesmo na ocorrência de alterações sensitivas e motoras [28], concordando com a observância de outros autores que não detectaram alterações durante a palpação dos nervos dos membros superiores e inferiores na maioria dos pacientes investigados [18].

Tendo em vista que o dano neural é considerado decisivo para o desencadeamento das incapacidades, sendo responsável pelas alterações na função sensitiva e/ou motora e estando subjacente a todas as formas da hanseníase [29], atenta-se também para a hipótese de falhas na qualificação profissional para a avaliação diagnóstica.

A baixa qualificação para identificar os sinais e sintomas da doença oportuniza 0 surgimento de sequelas. Neste sentido, destaca-se a necessidade de incentivar a implementação de políticas públicas, com o propósito de obter melhores resultados na qualidade da assistência [30] com ênfase para a realização do diagnóstico precoce e tratamento adequado dos doentes, consideradas melhores estratégias para prevenir a evolução da doença [31].

A baixa qualificação profissional é reflexo da falta de incentivos governamentais para o controle da hanseníase e para prevenção de incapacidades, que se revela esparsa no cenário atual [32]. Salienta-se ainda que não foram encontrados nos prontuários registros de algumas 
avaliações de rotina preconizadas pela OMS, em virtude da falta de materiais de baixo custo destinados a este fim.

$\mathrm{Na}$ análise do período de 2009 a 2014, a diferença encontrada para o GIF ( $p=0,038)$ justifica-se pela significativa diminuição da quantidade de pacientes diagnosticados com incapacidades de grau 2 (IG2) (2009: 23,2\% e 2014: 3,6\%) e aumento daqueles sem nenhum comprometimento nos sítios corporais (2009: 43,5\% e 2014: 63,6\%), o que sugere representar uma redução da magnitude da endemia.

A IG2 é um relevante indicador epidemiológico de avaliação do Programa Nacional de Controle da Hanseníase, visto que é utilizado na mensuração da força de morbidade, magnitude e perfil epidemiológico da doença, além de avaliar a qualidade dos serviços de saúde prestados. É preconizado pelo MS que na avaliação clínica, em caso de identificação de GIF diferentes nos sítios corporais, seja considerado o grau máximo, que varia de 0 a 2 [33].

Em comparação aos países asiáticos, o Brasil possui menores proporções de IG2 [2], o que pode ser justificado pela ampliação da assistência na atenção primária em saúde proporcionando melhora dos serviços relacionados ao controle da doença [31].

Em estudo realizado em São Luís/MA entre os anos de 2008-2009, verificou-se porcentagem de $42,1 \%$ de indivíduos apresentando incapacidades físicas já no momento do diagnóstico (GIF 1=29,82\% e GIF 2=12,28\%), sugerindo-se a necessidade de abordagens mais específicas no tocante a medidas de tratamento e reabilitação para evitar o agravamento da enfermidade e propiciar o controle das sequelas [34]. Esses valores foram inferiores aos encontrados por nosso estudo para o mesmo ano.

No tocante aos sítios corporais, ocorreu aumento da quantidade de indivíduos sem comprometimentos de forma significativa apenas para as mãos e os olhos. Uma das premissas para o maior cuidado nestes sítios está pautada na facilidade de visualização destas áreas tanto pelo paciente quanto pelos demais indivíduos, tornando precoce a percepção de alterações na sensibilidade e/ou funcionalidade [14], diferentemente do que acontece com os pés.

De maneira geral, entre os anos avaliados ocorreu significativa melhora no GIF, menor acometimento dos olhos, mãos e nervos, embora o número de pacientes diagnosticados entre as formas brandas da doença não tenha aumentado. Sob este prisma, hipotetiza-se que apesar da hanseníase permanecer ativa na região metropolitana de João Pessoa/PB, a assistência realizada na atenção secundária à saúde tem se mostrado satisfatória, no que tange a minimização dos agravos e prevenção de incapacidades.

O perfil sociodemográfico e clínico de pacientes acometidos pela hanseníase na região metropolitana de João Pessoa/PB foi caracterizado pelo sexo masculino, faixa etária economicamente ativa, baixa escolaridade, classificação multibacilar, forma clínica dimorfa, GIF 0 , pés como o sítio corporal mais comprometido e sem nervos afetados.

A análise indica redução da magnitude da endemia entre os anos de 2009 a 2014, apoiada pela redução de pacientes diagnosticados com IG2 e aumento daqueles sem comprometimentos na face, mãos e pés ou nervos afetados, evidenciando também a efetividade da assistência realizada no cenário da pesquisa, serviço de atenção secundária à saúde.

A assistência ofertada no serviço de atenção especializada proporciona aos indivíduos maiores oportunidades de tratamento e distância do seu local de moradia, tendo em vista o estigma e o preconceito associado a doença. Estes fatores contribuem para a manutenção destes no centro de referência, mesmo quando a indicação sugere sejam contra referenciados.

Essa premissa atua aumentando a carga de trabalho e contraria a orientação de descentralização preconizada para a assistência ao paciente acometido pela hanseníase, superlota o serviço, e inviabiliza que mais indivíduos com maiores necessidades sejam atendidos devidamente.

Salienta-se ainda que o nível de atenção secundária não responde pela busca ativa de casos, assim sendo, atende aos pacientes de demanda espontânea ou referenciados pela atenção primária. É compreensível, portanto, que muitos indivíduos sejam diagnosticados com algum acometimento tardio da doença, embora, no presente estudo, casos com incapacidades físicas tenham apresentado tendência de declínio entre os anos avaliados.

A coleta de dados foi realizada em fonte secundária de informação. Em vista disso, atentou-se para a existência de lacunas no preenchimento de informações e divergências entre 
os achados da avaliação clínica e classificação do grau de incapacidade, fatores limitantes ao estudo.

A relevância dos achados encontrados neste estudo, para a saúde pública e para a enfermagem, está pautada no conhecimento do comportamento sociodemográfico e clínico de pacientes acometidos pela hanseníase na região metropolitana de João Pessoa/PB para que ações possam ser realizadas de forma direcionada a esses grupos de indivíduos, visando a prevenção, diagnóstico, tratamento e reabilitação das sequelas físicas ocasionadas pela doença.

Sugere-se ainda a ampliação do escopo metodológico deste estudo, bem como a sua realização em outros estados e municípios, com vistas a proporcionar um monitoramento dos progressos alcançados no processo de erradicação da doença no país.

Referências

1. World Health Organization (WHO). Global leprosy update, 2014: need for early case detection. Weekly Epidemiol Rec 2015;36(90):461-76.

2. World Health Organization (WHO). Global leprosy update, 2015: time for action, accountability and inclusion. Weekly Epidemiol Rec 2016;35(91):405-20.

3. Ministério da Saúde (BR). Secretaria de Vigilância em Saúde. Situação epidemiológica da hanseníase no Brasil - análise de indicadores selecionados na última década e desafios para eliminação. Bol Epidemiol 2013;44(11):1-12.

4. Ribeiro GC, Fabri ACOC, Amaral EP, Machado IE, Lana FCF. Estimativa da prevalência oculta da hanseníase na microrregião de Diamantina - Minas Gerais. Rev Eletr Enfem 2014;16(4):728-35.

5. Departamento de Informática do SUS. Coeficiente de detecção de casos novos da hanseníase na Região Nordeste. 2015. [citado 2017 Jan 26]. Disponível em: http://tabnet.datasus.gov.br/cgi/tabcgi.exe?sinannet/hanseniase/cnv/hanswuf.def

6. Brito KKG, Andrade SSC, Santana EMF, Peixoto VB, Nogueira JA, Soares MJGO. Epidemiological analysis of leprosy in an endemic state of northeastern Brazil. Rev Gaúcha Enferm 2015;36(Spe):24-30.

7. Departamento de Informática do SUS. Coeficiente de detecção casos novos segundo Município no Estado da Paraíba. 2015. [citado 2017 Jan 26]. Disponível em: http://tabnet.datasus.gov.br/cgi/tabcgi.exe?sinannet/hanseniase/cnv/hanswpb.def

8. Ministério da Saúde. Secretaria de Vigilância em Saúde. Departamento de Vigilância de Doenças Transmissíveis. Plano integrado de ações estratégicas de eliminação da hanseníase, filariose, esquistossomose e oncocercose como problema de saúde pública, tracoma como causa de cegueira e controle das geohelmintíases: plano de ação 2011-2015. Brasília: Ministério da Saúde; 2012.

9. Ministério da Saúde. Secretaria de Vigilância em Saúde. Hanseníase, verminoses e tracoma têm cura: a experiência de uma campanha integrada. Bol Epidemiol 2016;47(21):1-10.

10. Ministério da Saúde (BR). Secretaria de Vigilância em Saúde. Departamento de Doenças Transmissíveis. Diretrizes para vigilância, atenção e eliminação da hanseníase como problema de saúde pública. Manual Técnico Operacional. Brasília: Ministério da Saúde; 2016.

11. Ministério da Saúde (BR). Secretaria de Vigilância em Saúde. Departamento de Vigilância Epidemiológica. Manual de prevenção de incapacidades. Brasília: Ministério da Saúde; 2008

12. Santos EJ, Paciencia GP, Urpia CC. Caracterização do perfil dos pacientes diagnosticados com hanseníase no município de Vilhena - Rondônia. Rev Rede Cuidados em Saúde 2016;10(3):1-16.

13. Lima MM, Aguilar AMM. Perfil epidemiológico da hanseníase em um município de Minas Gerais: Uma análise retrospectiva. Rev Pre Infec e Saúde 2015;1(3):1-9.

14. Barbosa DRM, Araújo AA, Damaceno JCF, Almeida MG, Santos AG. Perfil epidemiológico da hanseníase em cidade hiperendêmica do maranhão, 2005-2012. Rev Rede Cuidados Saúde 2014;8(1):1-12.

15. Kil AKA, Silvestre CM, Kaminice LM, Quintino LB, Lima LB, Paranhos MB et al. Deficiências e incapacidades por hanseníase: avaliação clínica e epidemiológica dos pacientes atendidos em um Centro de Referência Nacional do Brasil. Hansen Int 2012;37(1):25-33. 
16. Brito KKG, Araújo DAL, Uchôa REMN, Ferreira JDL, Oliveira MJGO, Lima JO. Epidemiology of leprosy in a state of northeast Brazil. Rev Enferm UFPE on line 2014;8(8):2686-93.

17. Faria CRS, Fregonesi CEPT, Coraza DAG, Andrade DM, Mantovani NADT, Silva JR, Mantovani AM. Grau de incapacidade física de portadores de hanseníase: estudo de coorte retrospectivo. Arq Ciênc Saúde 2015;22(4):58-62.

18. Mesquita R, Melo LTM, Vasconcelos RS, Soares DM, Félix GAA, Férrer LPA, Abdon APV. Neurofunctional evaluation in patients affected by leprosy. Rev Bras Promoç Saúde 2014;27(2):247-55.

19. Sarmento APA, Pereirão AM, Ribeiro F, Castro JL, Almeida MB, Ramos NM. Perfil epidemiológico da hanseníase no período de 2009 a 2013 no município de Montes Claros (MG). Rev Soc Bras Clin Med 2015;13(3):180-4.

20. World Health Organization (WHO). Leprosy today. [Internet]. 2010 [citado 2016 Out 24]. Disponível em: http://www.who.int/lep/transmission/en/index5.html

21. Lanza FM, Cortez DN, Gontijo TL, Rodrigues JSJ. Perfil epidemiológico da hanseníase no município de Divinópolis, Minas Gerais. Rev Enferm UFSM 2012; 2(2):365-74.

22. Aquino CMF, Rocha PAA, Guerra MCG, Coriolano MWL, Vasconcelos EMR, Alencar EN. Peregrinação (Via Crucis) até o diagnóstico da hanseníase. Rev Enferm UERJ 2015;3(2):185-90.

23. Ribeiro GC, Lana FCF. Incapacidades físicas em hanseníase: caracterização, fatores relacionados e evolução. Cogitare Enferm 2015;20(3):496-503.

24. Ribeiro Júnior AF, Vieira MA, Caldeira AP. Perfil epidemiológico da hanseníase em uma cidade endêmica no Norte de Minas Gerais. Rev Bras Clin Med 2012;10(4):272-7.

25. Brito AL, Monteiro LD, Ramos Júnior ANR, Heukelbach J, Alencar CH. Temporal trends of leprosy in a Brazilian state capital in Northeast Brazil: epidemiology and analysis by join points, 2001 to 2012. Rev Bras Epidemiol 2016;19(1):194-204.

26. Pinheiro MGC, Miranda FAN, Simpson CA, Vitor AF, Lira ALBC. Limitações e incapacidades físicas no pós-alta em hanseníase: uma revisão integrativa. Rev Baiana Enfem 2016;30(2):1-11.

27. Nardi SMT, Paschoal VD, Chiaravalloti-Neto F, Zanetta DMT. Leprosy-related disabilities after release from multidrug treatment: prevalence and spatial distribution. Rev Saúde Pública 2012;46(6):969-77.

28. Leite VMC, Lima JWO, Gonçalves HS. Neuropatia silenciosa em portadores de hanseníase na cidade de Fortaleza, Ceará, Brasil. Cad Saúde Pública 2011;27(4):65965.

29. Ribeiro FS, Silva MLA, Mendonça ALB, Soares JSA, Freitas CSL, Linhares MSC. Qualidade dos serviços prestados pelos centros de saúde da família de sobral - Ceará aos portadores de hanseníase nos anos de 2009 a 2010. Sanare 2012;11(2):44-51.

30. Monteiro LD, Alencar CHM, Barbosa JC, Braga KP, Castro MD, Heukelbach J. Incapacidades físicas em pessoas acometidas pela hanseníase no período pós-alta da poliquimioterapia em um município no Norte do Brasil. Cad Saúde Pública 2013;29(5):909-20.

31. Araújo AERA, Aquino DMC, Goulart IMB, Pereira SRF, Figueiredo IA, Serra HM, Fonseca PCA, Caldas AJM. Neural complications and physical disabilities in leprosy in a capital of northeastern Brazil with high endemicity. Rev Bras Epidemiol 2014;17(4):899-910.

32. Gonçalves A. Realities of leprosy control: updating scenarios. Rev Bras Epidemiol 2013;16(3):611-21.

33. Ministério da Saúde (BR). Portaria no 3.125, de 7 de outubro de 2010. Aprova as Diretrizes para Vigilância, Atenção e Controle da Hanseníase. Brasília: Ministério da Saúde; 2010.

34. Pacheco MAB, Aires MLL, Seixas ES. Prevalência e controle de hanseníase: pesquisa em uma ocupação urbana de São Luís, Maranhão, Brasil. Rev Bras Med Fam Comunidade 2014;9(30):23-30. 